\title{
ON MULTIPLICATION ALGEBRAS
}

\author{
BY
}

DAVID R. FINSTON

\begin{abstract}
The basic properties of multiplication algebras of nonassociative algebras over rings are introduced, including a discussion of multiplication algebras of tensor products of algebras. A characterization of semisimple artinian multiplication algebras is given along with a discussion of the nature of the simple factors of a multiplication algebra modulo its Jacobson radical. A criterion distinguishing the multiplication algebras of certain associative algebras is proved. Examples are included to illustrate certain proved results.
\end{abstract}

I. Introduction. Let $R$ be a commutative, associative ring with 1 . A binary $R$ algebra is a pair $(A, \mu)$, where $A$ is an $R$ module and $\mu: A \otimes_{R} A \rightarrow A$ is an $R$ module homomorphism. We will suppress the homomorphism $\mu$ and refer to the $R$ algebra $A ; \mu(a \otimes b)$ will be written $a b$. The associative $R$ subalgebra of the algebra of $R$ endomorphisms of $a$ generated by the endomorphisms $\lambda_{a}: c \rightarrow a c, \rho_{b}: c \rightarrow c b$ for all $a, b$ in $A$, is denoted $\mathscr{M}_{R}^{*}(A)$ and referred to as the multiplication ideal of $A$. The subalgebra of $\operatorname{End}_{R}(A)$ generated by $\mathscr{M}_{R}^{*}(A)$ and id ${ }_{A}$ is called the multiplication algebra of $A$ and is denoted $\mathscr{M}_{R}(A)$. Clearly, $\mathscr{M}_{R}(A)$ is a two sided ideal in $\mathscr{M}_{R}(A)$. We will suppress the subscript $R$ if no ambiguity arises.

Jacobson [6] initiated a study of multiplication algebras of algebras of finite dimension over a field. His main result in that context uncovered a striking relationship between the structure of the associative unital algebra $\mathscr{M}(A)$ and the not necessarily associative algebra $A$.

(1.1) TheOREM (JACOBSON). A necessary and sufficient condition that an algebra $A$ be isomorphic to a direct product of simple algebras is that $\mathscr{M}(A)$ be semisimple.

This theorem is included in Theorem (2.7).

Albert [1] made use of (1.1) to propose a definition for the radical of an algebra of finite dimension over a field. Defining the radical of an algebra to be the intersection of all ideals for which the quotient algebra is a product of simple algebras, the following theorem was obtained.

(1.2) THEOREM (ALBERT). Let $A$ be a finite dimensional algebra with a semisimple quotient, and let $J$ denote the Jacobson radical of $\mathscr{M}(A)$. Then either $A / J A$ is isomorphic to a product of simple algebras and $J A$ is the radical of $A$, or $A / J A$ is isomorphic to a product of simple algebras and a null algebra. In the latter case,

Received by the editors April 12, 1985.

1980 Mathematics Subject Classification. Primary 17A99.

(C)1986 American Mathematical Society $0002-9947 / 86 \$ 1.00+\$ .25$ per page 
$A / J A \cong S \Pi N$ with $S$ isomorphic to a product of simple algebras and $N$ null; the radical of $A$ is $J A+q^{-1}(N)$, where $q: A \rightarrow A / J A$ is the quotient homomorphism.

Müller [7] used the notion of separability for associative algebras over commutative rings to define separability for arbitrary algebras finitely generated and projective as modules over commutative rings.

(1.3) TheOREM (MÚlLeR). The multiplication algebra of a separable associative algebra is again separable.

Röhrl [8], Wisbauer [9] and Finston [4] have made extensive use of the multiplication algebra in their investigations of general nonassociative algebras. Farrand [2] studied the multiplication algebra in terms of its module action (see $\S I I$ below).

In this note, we begin a systematic study of multiplication algebras with the eventual goal of characterizing their structure. The main results we obtain are a characterization of all finite dimensional semisimple multiplication algebras, and a condition necessary for an associative algebra to be the multiplication algebra of an associative algebra. Examples are given to show that not all multiplication algebras arise as multiplication algebras of associative algebras.

II. Preliminary notions. The $R$ algebra $A$ has the structure of a left $\mathscr{M}(A)$ module whose submodules are exactly the two sided ideals of $A$. For an ideal $I$ of $A$, $(I: A)=\{\tau \in \mathscr{M}(A): \tau(A) \subset I\}$ is a two sided ideal of $\mathscr{M}(A)$. If $\mathscr{I}$ is a left ideal of $\mathscr{M}(A)$, then $\mathscr{I}(A)=\{\tau(a): \tau \in \mathscr{I}, a \in A\}$ is a two sided ideal of $A$. The following are some easy consequences of the definitions:

(2.1) (1) $I \subset J \rightarrow(I: A) \subset(J: A)$,

(2) $\mathscr{I} \subset J \rightarrow \mathscr{I}(A) \subset \mathscr{J}(A)$,

(3) $(I: A)(A) \subset I$,

(4) $(\mathscr{I}(A): A) \supset \mathscr{I}$,

(5) $\mathscr{I}(A) \subset(\mathscr{I}(A): A)(A) \subset \mathscr{I}(A)$; hence $\mathscr{I}(A)=(\mathscr{I}(A): A)(A)$

(6) $(I: A) \supset((I: A)(A): A) \supset(I: A)$; hence $(I: A)=((I: A)(A): A)$,

(7) $\mathscr{M}(A / I) \cong \mathscr{M}(A) /(I: A)$.

We will call an $R$ algebra simple if its only proper ideal is the zero ideal. Note that with this definition, a one dimensional algebra in which all products are 0 (i.e. a null algebra) is simple.

If $A$ is an algebra over the commutative ring $S$ and $R$ is a subring of $S$, then $A$ carries the structure of an $R$ algebra. Clearly, $\mathscr{M}_{S}^{*}(A)=\mathscr{M}_{R}^{*}(A)$; however, $\mathscr{M}_{S}(A)$ $=\mathscr{M}_{R}(A)$ if and only if $S$ is a subring of $\mathscr{M}_{R}(A)$.

An important instance of the above occurs when the centroid $Z(A)$, defined by $Z(A)=\operatorname{End}_{\mathscr{M}(A)}(A)$, is commutative; in this case $A$ has the structure of a $Z(A)$ algebra, and $R \subset Z(A)$. Example 1 of $\S I V$ shows that $\mathscr{M}_{Z(A)}(A)$ need not equal $\mathscr{M}_{R}(A)$ even when $Z(A)$ is commutative. The next lemma and its corollary are well known (e.g. Herstein [5], p. 46), although the formulation as been modified slightly.

(2.2) Lemma. Let $A$ be an $R$ algebra and $Z$ the centroid of $A$. Then $Z$ commutes on $A^{2}$. In particular, if $\operatorname{Hom}_{\mathscr{M}(A)}\left(A / A^{2}, A\right)=(0)$, then $Z(A)$ is commutative. 
Proof. Let $\tau, \psi \in Z(A), a, b \in A$. Then

$$
\tau \psi(a b)=\tau\left(\psi \circ \rho_{b}(a)\right)=\tau \rho_{b} \circ \psi(a)=\tau \psi(a) b=\tau \circ \lambda_{\psi(a)} b=\psi(a) \tau(b) .
$$

Similarly,

$$
\tau \psi(a b)=\tau \psi \circ \lambda_{a} b=\tau(a) \psi(b)=\psi \tau(a b) \text { by the previous computation. }
$$

The centroid of any unital or simple algebra is thus commutative, as is the centroid of any algebra $A$ satisfying $A^{2}=A$, or $\mathscr{M}^{*}(A)=\mathscr{M}(A)$ (since $A^{2}=$ $\left.\mathscr{M}^{*}(A) A\right)$.

(2.3) Corollary. If $A$ is a simple $R$ algebra, then $Z(A)$ is a commutative field.

Proof. Use Lemma 2.2 and Schur's lemma.

Formation of the multiplication algebra is not a functor on $R$ algebras, as the following example shows. Consider $\mathbf{C}$, the field of complex numbers, and $\mathbf{H}$, the algebra of real quaternions as algebras over $\mathbf{R}$, the field of real numbers. Embed $\mathbf{C} \rightarrow \mathbf{H}$ via $a+b i \rightarrow a+b i$, where $i^{2}=-1$ and $1, i, j, k$ is the usual basis for $\mathbf{H}$. Since $\lambda_{i}-p_{i}$ doesn't vanish on $\mathbf{H}$, there is no natural extension of the embedding $\mathbf{C} \rightarrow \mathbf{H}$ to a homomorphism $\mathscr{M}_{R}(\mathbf{C}) \rightarrow \mathscr{M}_{R}(\mathbf{H})$. Nevertheless, the multiplication algebra does respect certain constructions and homomorphisms.

(2.4) Lemma. Let $f: A \rightarrow B$ be a surjective homomorphism of $R$ algebras. Then $f$ induces a surjective homomorphism $\tilde{f}: \mathscr{M}_{R}(A) \rightarrow \mathscr{M}_{R}(B)$.

Proof. Set $f\left(\lambda_{a}\right)=\lambda_{f(a)}$ and $f\left(\rho_{b}\right)=\rho_{f(b)}$ for all $a, b$ in $A$. If the extension to $\mathscr{M}(A)$ is well defined, then surjectivity will follow from surjectivity of $f$.

Suppose $\Phi$ is a sum of compositions of right and left multiplications representing 0 in $\operatorname{End}_{R}(A)$. Let $b=f(a) \in B$. Since $f$ is a homomorphism, $0=f(\Phi(a))=$ $\tilde{f}(\Phi)(b)$.

The assertion of part (a) of the next lemma appears in [7] without proof. The proofs of parts (b) and (c) rely on the notion of a projective basis for a finitely generated projective module whose definition is recalled here. A projective basis $\left\{f_{i}, \dot{x}_{i} \mid i \leqslant j \leqslant n\right\}$ for an $R$ module $M$ consists of a collection of $R$ module homomorphisms $f_{i} \in \operatorname{Hom}_{R}(M, R)$ and elements $x_{i} \in M$ satisfying $x=$ $\sum_{i=1}^{n} f_{i}(x) x_{i}$ for all $x \in M$. Necessary and sufficient for $M$ to be a projective $R$ module is the existence of a projective basis.

(2.5) Lemma. Let $A$ and $B$ be $R$ algebras finitely generated and projective as $R$ modules, and let $S$ be a commutative associative, unital $R$ algebra.

(a) The homomorphism $h: A \rightarrow S \otimes_{R} A$ given by $a \rightarrow 1 \otimes$ a induces a homomorphism $\tilde{h}: \mathscr{M}(A) \rightarrow S \otimes_{R} \mathscr{M}(A)$. Moreover, $S \otimes \mathscr{M}_{R}(A) \cong \mathscr{M}_{S}\left(S \otimes_{R} A\right)$.

(b) The inclusions $\mathscr{M}_{R}(A) \rightarrow \operatorname{End}_{R}(A)$ and $\mathscr{M}_{R}(B) \rightarrow \operatorname{End}_{R}(B)$, and the isomorphism $\operatorname{End}_{R}(A) \otimes_{R} \operatorname{End}_{R}(B) \cong \operatorname{End}_{R}\left(A \otimes_{R} B\right)$ induce an algebra homomorphism $h$ : $\mathscr{M}_{R}(A) \otimes_{R} \mathscr{M}_{R}(B) \rightarrow \operatorname{End}_{R}\left(A \otimes_{R} B\right)$.

There is an injective $R$ algebra homomorphism

$$
\hat{h}: \mathscr{M}_{R}^{*}\left(A \otimes_{R} B\right) \rightarrow \operatorname{im}\left(\left.h\right|_{\mathscr{M}_{R(A)}^{*} \otimes \mathscr{M}_{R(B)}^{*}}\right) .
$$


(c) The map $\hat{h}$ in $(b)$ extends to an injective homomorphism $\mathscr{M}_{R}(A \otimes B) \rightarrow \operatorname{im}(h)$, which is an isomorphism if $\mathscr{M}_{R}^{*}(A)=\mathscr{M}_{R}(A), \mathscr{M}_{R}^{*}(B)=\mathscr{M}_{R}(B)$, and either $A$ or $B$ has a unit element.

Proof. (a) The homomorphism $\mathscr{M}_{R}(A) \rightarrow S \otimes_{R} \mathscr{M}_{R}(A)$ is given by $\Phi \rightarrow 1_{S} \otimes \Phi$.

Following with the isomorphisms $S \otimes_{R} \operatorname{End}_{R}(A) \cong \operatorname{End}_{S}\left(S \otimes_{R} A\right)$, we obtain an injection $S \otimes_{R} \mathscr{M}(A) \rightarrow \mathscr{M}\left(S_{S} \otimes_{R} A\right)$. That this map is also surjective follows by observing that

$$
s \otimes \lambda_{a} \rightarrow \lambda_{s \otimes a}, \quad s \otimes \rho_{a} \rightarrow \rho_{s \otimes a}, \quad 1_{S \otimes A} \mathrm{id} \rightarrow \mathrm{id}_{S \otimes A}
$$

which generate $\mathscr{M}_{S}(S \otimes A)$.

(b) We follow an element of $\mathscr{M}_{R}^{*}\left(A \otimes_{R} B\right)$ along the standard isomorphism $\operatorname{End}_{R}\left(A \otimes_{R} B\right) \rightarrow \operatorname{End}_{R}(A) \otimes_{R} \operatorname{End}_{R}(B)$.

Let $\left\{f_{i}, x_{i}\right\},\left\{g_{j}, y_{j}\right\}$ be projective bases for $A$ and $B$, respectively. Set $\pi_{j}$ : $A \otimes_{R} B \rightarrow A \otimes_{R}$ End $_{R} B$ to be the map

$$
\pi_{j}: a \otimes b \rightarrow a \otimes g_{j}(\quad) \cdot b .
$$

Set $\theta_{i}: A \otimes_{R}$ End $_{R} \rightarrow \operatorname{End}_{R} A \otimes$ End $B_{R}$ to be the map

$$
\theta_{i}: a \otimes h \rightarrow f_{i}(\quad) \cdot a \otimes h .
$$

The isomorphism is then given, for $\Phi \in \operatorname{End}_{R}(A \otimes B)$ by

$$
\Phi \rightarrow \sum_{i, j} \theta_{i}\left(\pi_{j} \circ \Phi\left(x_{i} \otimes y_{j}\right)\right) \text {. }
$$

Observe that, for $a \in A, \lambda_{a}=\sum_{i} f_{i}(\quad) a x_{i}$ and, for $b \in B, \lambda_{b}=\sum_{j} g_{j}(\quad) \cdot b y_{j}$. Under the above isomorphism,

$$
\begin{aligned}
\lambda_{a \otimes b} & \rightarrow \sum_{i, j} \theta_{i}\left(\pi_{j}\left(a x_{i} \otimes b y_{j}\right)\right) \\
& =\sum_{i, j} \theta_{i}\left(a x_{i} \otimes g_{j}(\quad) b y_{j}\right) \\
& =\sum_{i, j} f_{i}(\quad)\left(a x_{i} \otimes g_{j}(\quad) b y_{j}\right) \\
& =h\left(\lambda_{a} \otimes \lambda_{b}\right) .
\end{aligned}
$$

Similarly, $\rho_{a \otimes b} \rightarrow h\left(\rho_{a} \otimes \rho_{b}\right)$.

Extending the map in (a) to $\mathscr{M}(A \otimes B)$, observe that if $\mathscr{M}^{*}(A)=\mathscr{M}(A)$ and $\mathscr{M}^{*}(B)=\mathscr{M}(B)$, and $A$ or $B$ has a unit, then $\mathrm{id}_{A} \otimes \tau$ and $\phi \otimes \mathrm{id}_{B}$ are in the image for all $\tau \in \mathscr{M}(B)$ and $\phi \in \mathscr{M}(A)$.

Example 4.4 provides a simple, finite dimensional algebra $A$ for which

$$
\hat{h}(\mathscr{M}(A \otimes A)) \subsetneq \operatorname{im}(h) \text {. }
$$

(2.6) LemMA. Let $\left\{A_{i} \mid 1 \leqslant i \leqslant n\right\}$ be a finite collection of $R$ algebras. Then

(a) $\mathscr{M}_{R}^{*}\left(\prod_{i=1}^{n} A_{i}\right) \cong \prod_{i=1}^{n} \mathscr{M}_{R}^{*}(A)$.

(b) If $\mathscr{M}_{R}^{*}\left(A_{i}\right)=\mathscr{M}_{R}\left(A_{i}\right)$ for at least $n-1 A_{i}$, then

$$
\mathscr{M}_{R}\left(\prod_{i=1}^{n} A\right) \cong \prod_{i=1}^{n} \mathscr{M}_{R}\left(A_{i}\right)
$$


(c) Conversely, if $\mathscr{A} \cong \prod_{i=1}^{t} \mathscr{A}_{i}$, an associative unital $R$-algebra, is isomorphic to the multiplication algebra of an $R$ algebra $A$, then $A \cong\left(\prod_{i=1}^{s} A_{i}\right) \prod N$, where $t-1 \leqslant$ $s \leqslant t, N$ is a null algebra, and $\mathscr{A}_{i}=\mathscr{M}\left(A_{i}\right)$ for $1 \leqslant i \leqslant s$.

Proof. To prove assertion (a) it suffices to consider $n=2$. The projections $A \Pi B \rightarrow A$ and $A \Pi B \rightarrow B$ induce surjections $\mathscr{M}^{*}(A \Pi B) \rightarrow \mathscr{M}^{*}(A)$ and $\mathscr{M}^{*}(A \Pi B) \rightarrow \mathscr{M}^{*}(B)$, which in turn induce $\mathscr{M}^{*}(A \Pi B) \rightarrow \mathscr{M}^{*}(A) \Pi \mathscr{M}^{*}(B)$. The latter is easily seen to be an isomorphism.

Assertion (b) follows from the observation that for at least $n-1 A_{i}, \mathrm{id}_{A_{i}} \in$ $\mathscr{M}^{*}\left(A_{i}\right)$. Their images in $\left.\mathscr{M}^{*}(\Pi A)_{i}\right)$ together with $\operatorname{id}_{\Pi_{i} A_{i}} \in \mathscr{M}\left(\Pi A_{i}\right)$ yield the image of id $A_{i} \in \mathscr{M}\left(\Pi A_{i}\right)$ for all $i \leqslant i \leqslant n$.

To prove the converse, (c), assume $\mathscr{M}_{R}(A) \cong \prod_{i=1}^{t} U_{i}$. Then

$$
A=\mathscr{M}_{R}(A) A=\left(\prod_{i=1}^{t} U_{i}\right) A=\prod_{i=1}^{t}\left(U_{i} A\right)
$$

since the $U_{i} A$ are orthogonal ideals of $A$.

Let $A_{i}=U_{i} A$, and set $N=\prod\left\{A_{i} \mid A_{i}^{2}=(0)\right\}$. After a renumbering, we obtain $A \cong\left(\prod_{i=1}^{s} A_{i}\right) \Pi N$. Denote by $I_{j}$ the image of $\left(\prod_{i \neq j} A_{i}\right) \Pi N$ in $A$. Then $A_{j} \cong$ $A / I_{j}$, and

$$
\begin{aligned}
\mathscr{M}\left(A_{j}\right) & \cong \mathscr{M}(A) /\left(I_{j}: A\right) \cong \prod U_{i} /\left(\prod_{i \neq j} U_{i} A: A\right) \\
& \cong \prod U_{i} / \prod_{i \neq j}\left(U_{i} A: A\right) \cong U_{j} . \square
\end{aligned}
$$

(2.7) THEOREM. The multiplication algebra of a finitely generated $R$ algebra is semisimple artinian if and only if $R$ is a product of fields and $A$ is isomorphic to a finite product of simple $R$ algebras. Moreover, writing $A=A_{s} \Pi N$, where $A_{s}^{2}=A_{s}, N$ is null, and $K \cong R /$ Ann $N$, we have $\mathscr{M}_{R}(A) \cong \mathscr{M}_{R}\left(A_{s}\right) \Pi K$, and $\mathscr{M}_{R}\left(A_{s}\right)$ is isomorphic to a product of full matrix rings over commutative fields.

Proof. Assuming $\mathscr{M}_{R}(A)$ semisimple artinian, we obtain that $R$ is artinian from the Wedderburn-Artin theorem and the fact that $R$ is a commutative subring of $\mathscr{M}_{R}(A)$. Semisimplicity of $R$ follows by observing that a nilpotent ideal in $R$ extends to a nilpotent ideal in $\mathscr{M}_{R}(A)$. Given the structure of $R$ and Lemma (2.6), we may assume $R$ is a field and reduce to showing that $\mathscr{M}_{R}(A)$ is simple artinian if and only if $A$ is simple with $A^{2} \neq(0)$, or $A$ is null. In the latter case, $\mathscr{M}_{R}(A) \cong R$.

Suppose $\mathscr{M}_{R}(A)$ is simple artinian. As a left $\mathscr{M}_{R}(A)$ module then, $A$ decomposes as a finite direct sum of simple $\mathscr{M}_{R}(A)$ submodules $A_{i}, 1 \leqslant i \leqslant n$. These, in turn, are simple, orthogonal ideals of $A$, and hence simple, orthogonal subalgebras of $A$. By (2.5), $\mathscr{M}_{R}^{*}(A)=\prod_{i} \mathscr{M}_{R}^{*}\left(A_{i}\right)$, but simplicity of $\mathscr{M}_{R}(A)$ forces either $\mathscr{M}(A) \cong$ $\mathscr{M}^{*}(A) \cong \prod_{i=1}^{n} \mathscr{M}^{*}\left(A_{i}\right)$ or $\mathscr{M}^{*}(A)=(0)$. In the latter case, $\mathscr{M}(A)=R$, and $A$ is null. In the former case, $n \leqslant 2$. If $n=1$, then $A$ is simple with $A^{2} \neq(0)$. If $n=2$, then $A \cong A \Pi N$, where $A^{2} \neq(0)$ and $N$ is null. Applying (2.6) again, we have $\mathscr{M}(A) \cong \mathscr{M}(A) \Pi R$, a contradiction.

To prove the converse, let $A$ be a finite dimensional simple algebra over a field $R$. If $A^{2}=(0)$, then $\mathscr{M}(A)=R$. Otherwise, $A^{2}=A$ and the centroid of $A$ is a 
commutative field. Since $A$ is a simple faithful module for $\mathscr{M}(A), \mathscr{M}(A)$ is primitive, hence semisimple, hence simple by (2.6). By the density theorem, $\mathscr{M}(A)$ $\cong \operatorname{End}_{Z(A)}(A)$.

III. Particular algebras. One consequence of Theorem (2.7) is that certain algebras cannot arise as multiplication algebras. Indeed, the inspiration for that result was a question posed by McCrimmon and Dorfmeister as to whether a divison algebra could arise as the multiplication algebra of a finite dimensional algebra. In this section, we investigate the interplay between the properties of a finite dimensional algebra and the simple factors of its multiplication algebra modulo the Jacobson radical. We begin with a second proof, due to Lance Small (personal communication), that noncommutative division rings do not occur as multiplication algebras. His argument has no dimensionality constraint.

(3.1) THEOREM. If a divison ring is a multiplication algebra, then it must be commutative.

Proof. Assume $D$ is a divison ring isomorphic to $\mathscr{M}(A)$. Then for any $a \in A$, $\lambda_{a}-\rho_{a}$ is either identically zero or invertible. However, $a$ is in the kernel of $\lambda_{a}-\rho_{a}$, so that $A$ is commutative.

Now observe that $\mathscr{M}(A)$ is also commutative. It suffices to show that $\lambda_{a} \circ \lambda_{b}=$ $\lambda_{b} \circ \lambda_{a}$ for all $a, b$ in $A$ since $\lambda_{a}=\rho_{a}$ for all $a \in A$.

$$
\lambda_{a} \circ \lambda_{b}(a)=a(b a)=a(a b)=(a b) a=\lambda_{a b}(a) .
$$

Thus $\lambda_{a} \circ \lambda_{b}=\lambda_{a b}$. Finally

$$
\lambda_{a b}=\lambda_{b a}=\lambda_{b} \circ \lambda_{a}=\lambda_{a} \circ \lambda_{b} .
$$

The following proposition extends Theorem (2.6).

(3.2) Proposition. Let $A$ be a finite dimensional algebra, and $J$ the Jacobson radical of $\mathscr{M}(A)$. If $J$ is a maximal ideal, then $\mathscr{M}(A) / J$ is isomorphic to a full matrix ring over a commutative field.

Proof. $\mathscr{M}(A) / J$ is a simple ring, and $J A$ is an ideal of $A$. Comparing ideals in $\mathscr{M}(A)$, we have $J \subset(J A: A) \subset \mathscr{M}(A)$. By maximality, $J=(J A: A)$, so that $\mathscr{M}(A / J A)=\mathscr{M}(A) /(J A: A)=\mathscr{M}(A) / J$. Thus $\mathscr{M}(A) / J$ is the multiplication algebra of a simple algebra. The result now follows from (2.7).

In view of (2.7) and (3.2) one suspects that the simple rings appearing as the factors modulo the Jacobson radical of the multiplication algebra of a finite dimensional algebra are rather special. Example 2 of $\S I V$ shows that matrix rings over division rings can arise in this context. The next series of arguments elucidates when this cannot occur.

Let $A$ be an $R$ algebra of finite length as an $\mathscr{M}(A)$ module. Then $A$ has a composition series of $\mathscr{M}(A)$ submodules (= ideals): $(0)=I_{0} \subset I_{1} \subset \cdots \subset I_{n}=A$. Each $I_{j} / I_{j-1}$ is a simple, faithful, and finitely generated module over $\mathscr{M}(A) /\left(I_{j-1}: I_{j}\right)$; as such $\mathscr{M}_{j}=\mathscr{M}(A) /\left(I_{j-1}: I_{j}\right)$ is primitive and $D_{j}=$ $\operatorname{End}_{\mathscr{M}(A)}\left(I_{j} / I_{j-1}\right)$ is a divison ring.

(3.3) LeMma. If $I_{j}^{2} \not \subset I_{j-1}$, then $D_{j}$ is commutative. 
Proof. The argument is an adaptation of the proof of (2.2).

Assuming $I_{j}^{2} \not \subset I_{j-1}$, simplicity of the $\mathscr{M}(A)$ module $I_{j} / I_{j-1}$ implies that it generated as an $\mathscr{M}(A)$ module by $\left(I_{j}^{2}+I_{j-1}\right) / I_{j-1}$. Let $\tau, \phi \in D_{j}, f \in \mathscr{M}_{j}$, and $\bar{a}$, $\bar{b} \in I_{j} / I_{j-1}$, with $\overline{a b} \neq 0$. Under these assumptions, $\lambda_{\bar{a}}$ and $\rho_{\bar{b}}$ can be identified with elements of $\mathscr{M}_{j}$, and we have

$$
\tau \psi f(\overline{a b})=f \tau \psi(\overline{a b})=f \tau \psi\left(\lambda_{\bar{a}}(\bar{b})\right)=f \tau \lambda_{\bar{a}} \psi(\bar{b})=f \tau[\bar{a} \psi(\bar{b})]=f \tau(\bar{a}) \psi(\bar{b}) .
$$

Alternatively,

$$
\tau \psi f(\overline{a b})=f \tau \psi\left(\rho_{\bar{b}}(\bar{a})\right)=f \tau[\psi(\bar{a}) \bar{b}]=f \psi(\bar{a}) \tau(\bar{b})=\psi \tau f(\overline{a b}) .
$$

Since $f, \bar{a}, \bar{b}$ were arbitrary, $\psi$ and $\tau$ commute on all of $I_{j} / I_{j \cdot 1}$.

Suppose, as in the lemma above, that the $R$ algebra $A$ has finite length as an $\mathscr{M}(A)$ module. For any composition series $(0)=I_{0} \subset I_{1} \subset \cdots \subset I_{n}=A$ we have $J \subset \operatorname{Ann}\left(I_{j} / I_{j-1}\right)=\left(I_{j-1}: I_{j}\right)$, where $J$ is the Jacobson radical of $\mathscr{M}(A)$. In case $\mathscr{M}(A)$ is artinian, none of the finitely many simple factors of $\mathscr{M}(A) / J$ annihilates every composition factor $I_{j} / I_{j-1}$, as $A$ is a faithful $\mathscr{M}(A)$ module. Thus each simple factor of $\mathscr{M}(A) / J$ is isomorphic to some $\mathscr{M}_{j}=\mathscr{M}(A) /\left(I_{j-1}: I_{j}\right)$. We will say that a composition factor $I_{j} / I_{j-1}$ has square zero if in the algebra $A / I_{j-1}$, the minimal ideal $I_{j} / I_{j-1}$ has square zero.

Lemma (3.3) and the above discussion prove

(3.4) Proposition. Let $A$ be a finite dimensional algebra over a field, and $J$ the Jacobson radical of $\mathscr{M}(A)$. If, in some composition series of ideals of $A$, no composition factor has square zero, then $\mathscr{M}(A) / J$ is isomorphic to a product of full matrix rings over commutative fields.

If $A$ is an associative $R$ algebra, the homomorphisms $A \stackrel{\lambda}{\rightarrow} \mathscr{M}^{*}(A) \stackrel{\rho}{\leftarrow} A^{\text {op }}$ given by $\lambda(a)=\lambda_{a}, \rho(a)=\rho_{a}$ induce a homomorphism $\varepsilon: A \otimes_{R} A^{\mathrm{op}} \rightarrow \mathscr{M}_{R}^{*}(A)$, which is easily seen to be surjective. This observation is exploited in the following theorem.

(3.5) THEOREM. Let $A$ be a finite dimensional associative algebra over a field $K$, and suppose that $A / J$ is a separable $K$ algebra, where $J$ is the Jacobson radical of $A$. Denote by $\mathscr{J}$ the Jacobson radical of $\mathscr{M}^{*}(A)$. Then either $\mathscr{J}$ is a maximal ideal, in which case $\mathscr{M}^{*}(A) / J$ is isomorphic to a full matrix ring over a commutative field, or at least two factors of $\mathscr{M}^{*}(A) / J$ are isomorphic to full matrix algebras over commutative fields.

Proof. The Wedderburn principal theorem asserts that $A$ has a separable subalgebra $S$ such that $A=S \oplus J$. Let $\varepsilon: A \otimes_{K} A^{\text {op }} \rightarrow \mathscr{M}^{*}(A)$, and observe that with respect to the Wedderburn decomposition of $A$, we have

$$
A \otimes_{K} A^{\mathrm{op}} \cong S \otimes_{K} S^{\mathrm{op}} \oplus A \otimes_{K} J^{\mathrm{op}} \oplus J \otimes_{K} A^{\mathrm{op}}
$$

Moreover, the image of $A \otimes J^{\mathrm{op}} \oplus J \otimes A^{\mathrm{op}}$ in $\mathscr{M}^{*}(A)$ is the Jacobson radical of that ring. Thus $\varepsilon$ induces a homomorphism $\hat{\varepsilon}: S \otimes_{K} S^{\text {op }} \rightarrow \mathscr{M}(A) / J$. Since all tensor products are over $K$, we will suppose the subscript in the remainder of the proof. 
The separability assumption on $S$ implies that $S \otimes S^{\mathrm{op}}$ is semisimple. In particular, $S \otimes S^{\text {op }}$ has a unit element, so that $\hat{\varepsilon}$ is surjective. Write $S=\prod_{i=1}^{t} R_{i}$, where the $R_{i}$ are simple separable $K$ algebras; we may assume $t>1$.

There is a composition series of ideals of $A$,

$$
(0) \subset J_{1} \subset J_{2} \cdots \subset J_{m}=J \subset I_{1} \subset \cdots \subset I_{t}=A,
$$

where $J_{i} \subset J$, and $I_{j}=I_{j-1}+R_{j}$. We examine the affect of $\hat{\varepsilon}\left(S \otimes S^{\mathrm{op}}\right)$ on the composition factors $I_{j} / I_{j-1}=R_{j}$.

Clearly, $\left(R_{i} \otimes R_{j}^{\mathrm{op}}\right) R_{k}=(0)$ unless $i=j=k$. Moreover, none of $I_{j} / I_{j-1}$ has square zero, so that $\mathscr{M}(A) /\left(I_{j-1}: I_{j}\right) \cong \operatorname{End}_{D_{j}}\left(I_{j} / I_{j-1}\right)$, where $D_{j}$ is a commutative field. Restricting $\left(R_{j} \otimes R_{j}^{\text {op }}\right)$ to $I_{j} / I_{j-1}$, we obtain $\operatorname{End}_{D_{j}}\left(I_{j} / I_{j-1}\right)$ as a simple

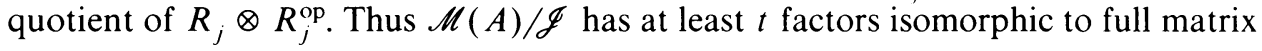
rings over commutative fields.

IV. Examples and concluding remarks. Examples (4.1)-(4.4) are finite dimensional algebras over a field. They are described as follows: a basis $e^{1}, \ldots, e^{d}$ is prescribed, and all products of basis elements are written down. The multiplication algebras are subalgebras of $d \times d$ matrices relative to the given basis. The convention is to describe an algebra of matrices by writing a typical element. For example, the algebra of two by two upper triangular matrices is described by $\left[\begin{array}{ll}a & b \\ 0 & c\end{array}\right]$. The radical of $\mathscr{M}(A)$ will always be denoted by $J$.

(4.1) An algebra $A$ whose centroid $Z$ is commutative, but for which $\mathscr{M}(A) \neq \mathscr{M}_{Z}(A)$ (i.e. $(Z / A) \not \subset \mathscr{M}(A))$.

$$
\begin{array}{cccc}
e^{1} e^{1}=0 & e^{2} e^{1}=0 & e^{3} e^{1}=0 & e^{4} e^{1}=0 \\
e^{1} e^{2}=0 & e^{2} e^{2}=0 & e^{3} e^{2}=0 & e^{4} e^{2}=0 \\
e^{1} e^{3}=e^{1} & e^{2} e^{3}=0 & e^{3} e^{3}=0 & e^{4} e^{3}=0 \\
e^{1} e^{4}=0 & e^{2} e^{4}=0 & e^{3} e^{4}=0 & e^{4} e^{4}=e^{2} \\
\mathscr{M}(A) & =\left[\begin{array}{llll}
0 & 0 & b & 0 \\
0 & b & 0 & c \\
0 & 0 & b & 0 \\
0 & 0 & 0 & b
\end{array}\right], & Z(A)=\left[\begin{array}{llll}
\alpha & 0 & 0 & 0 \\
0 & \alpha & \beta & 0 \\
0 & 0 & \alpha & 0 \\
0 & 0 & 0 & \alpha
\end{array}\right] .
\end{array}
$$

(4.2) An example of a necessarily nonassociative algebra $A$ for which $\mathscr{M}(A) / J$ has only one factor isomorphic to a matrix ring over a field. $A$ has basis $e^{1}, e^{2}, e^{3}, e^{4}, e^{5}$ over $\mathbf{R}$. The nonzero basis products are:

Observe that

$$
\begin{array}{ll}
e^{1} e^{5}=e^{2} & e^{5} e^{1}=e^{3} \\
e^{2} e^{5}=-e^{1} & e^{5} e^{2}=-e^{4} \\
e^{3} e^{5}=e^{4} & e^{5} e^{3}=-e^{1} \\
e^{4} e^{5}=-e^{3} & e^{5} e^{4}=e^{2}
\end{array}
$$

$$
e^{5}\left(e^{5} e^{1}\right)=e^{5} e^{3}=-e^{1}, \quad\left(e^{5} e^{5}\right) e^{1}=0 .
$$

Using $e_{i j}$ to denote the $i j$ th matrix unit, we have

$$
\begin{aligned}
& \lambda e_{5}=e_{25}, \quad \lambda e_{2}=-e_{15} \lambda e_{3}=e_{45}, \quad \lambda e_{4}=-e_{35}, \quad \lambda e_{5}=e_{31}-e_{42}-e_{13}+e_{24}, \\
& \rho e_{1}=e_{35}, \quad \rho e_{2}=-e_{45}, \quad \rho e_{3}=-e_{15}, \quad \rho e_{4}=e_{25}, \\
& \rho e_{5}=e_{21}-e_{12}+e_{43}-e_{34}, \quad-\left(\rho e_{5}\right)_{2}=e_{11}+e_{22}+e_{33}+e_{44} .
\end{aligned}
$$


Since id ${ }_{A} \in \mathscr{M}(A), e_{55} \in \mathscr{M}(A)$. Obvious matrix algebra shows that

$$
\mathscr{M}(A)=\left[\begin{array}{rrrrr}
a & c & d & e & f \\
-c & a & e & -d & g \\
-d & -e & a & c & h \\
-e & -d & -c & a & i \\
0 & 0 & 0 & 0 & b
\end{array}\right] .
$$

Note that

$$
\left[\begin{array}{lllll}
0 & 0 & 0 & 0 & \gamma \\
0 & 0 & 0 & 0 & \beta \\
0 & 0 & 0 & 0 & \gamma \\
0 & 0 & 0 & 0 & \gamma \\
0 & 0 & 0 & 0 & 0
\end{array}\right]
$$

is a nilpotent ideal modulo which $\mathscr{M}(A)$ is isomorphic to $\mathbf{H} \Pi \mathbf{R}$.

(4.3) An example of an associative algebra $A$ satisfying $\mathscr{M}(A) / J$ separable, and isomorphic to a product of two full matrix rings and a division ring. Let $A \cong(\mathbf{H} \Pi \mathbf{R})$ $\oplus \mathbf{N}$ be the Wedderburn decomposition where $\mathbf{N}=\oplus_{i=1}^{4} \mathbf{R} f^{i}, \mathbf{H}$ has basis $e^{1}, i, j$, $k, \mathbf{R}$ has basis $e^{2}$, and we identify these with their images in $A$. The multiplication in $A$ is given as follows:

$$
\begin{array}{llll}
f^{i} f^{j}=0 & 1 \leqslant i, j \leqslant 4 & e^{1} i=i=i e^{1} & i^{2}=j^{2}=k^{2}=-e^{1} \\
& & \begin{array}{l}
e^{1} j=j=j e^{1} \\
e^{1} k=k=k e^{1}
\end{array} & j i=k, j k=i, k i=j \\
e^{1} f^{i}=f^{i} & 1 \leqslant i \leqslant 4 & j i=-k j=-i, i k=-j \\
& & e^{1} e^{1}=e^{1} & \\
f^{i} e^{2}=f^{i} & 1 \leqslant i \leqslant 4 & e^{2} e^{2}=e^{2} \\
i f^{1}=f^{2} & j f^{1}=f^{3} & k f^{1}=f^{4} \\
i f^{2}=-f^{1} & j f^{2}=-f^{4} & k f^{2}=f^{3} \\
i f^{3}=f^{4} & j f^{3}=-f^{1} & k f^{3}=-f^{2} \\
i f^{4}=-f^{3} & j f^{4}=f^{2} & k f^{4}=-f^{1}
\end{array}
$$

All other products are 0 .

Note. $\mathbf{H} N \mathbf{R}=N, \mathbf{R} N \mathbf{H}=(0)$ so that

$$
\begin{aligned}
\mathscr{M}(A) & \cong\left(\mathbf{H} \otimes_{\mathbf{R}} \mathbf{H}^{\mathrm{op}} \Pi \mathbf{H} \otimes R_{\mathbf{R}}^{\mathrm{op}} \Pi \mathbf{R} \otimes_{\mathbf{R}} \mathbf{R}^{\mathrm{op}}\right) \otimes J \\
& \cong M_{4}(\mathbf{R}) \Pi \mathbf{R} \Pi \mathbf{H} \oplus J .
\end{aligned}
$$

(4.4) An example of a simple algebra $A$ over a field $K$ for which $\mathscr{M}(A) \cong \operatorname{End}_{K}(A)$, but $\mathscr{M}(A \otimes A) \supsetneqq$ End $_{K}(A \otimes A)$. Let $A$ be the two dimensional algebra over any field $K$ with basis $e^{1}, e^{2}$ and multiplication

$$
\begin{gathered}
e^{1} e^{1}=e^{2}, \quad e^{2} e^{1}=0, \quad e^{1} e^{2}=0, \quad e^{2} e^{2}=e^{1}, \\
\mathscr{M}(A)=\left[\begin{array}{ll}
a & b \\
c & d
\end{array}\right] .
\end{gathered}
$$

Setting

$$
f^{1}=e^{1} \otimes e^{1}, \quad f^{2}=e^{2} \otimes e^{2}, \quad f^{3}=e^{1} \otimes e^{2}, \quad f^{4}=e^{2} \otimes e^{1},
$$


$A \otimes A$ has multiplication

$$
f^{1} f^{1}=f^{2}, \quad f^{2} f^{2}=f^{1}, \quad f^{3} f^{3}=f^{4}, \quad f^{4} f^{4}=f^{3} ;
$$

all other products are zero. Relative to the basis $\left\{f^{1}, f^{2}, f^{3}, f^{4}\right\}$,

$$
\mathscr{M}(A \otimes A)=\left[\begin{array}{llll}
a & b & 0 & 0 \\
c & d & 0 & 0 \\
0 & 0 & e & f \\
0 & 0 & g & h
\end{array}\right] .
$$

The results in this note have the common theme of investigating a multiplication algebra through its simple factors modulo the Jacobson radical. One issue which remains to be addressed to the way the radical can lie inside a multiplication algebra.

Let $U$ be a finite dimensional unital associative algebra over the algebraically closed field $K$ with Wedderburn decomposition $U \cong S \oplus J$. Assume now that $U$ is embedded unitally as a subalgebra of $\mathscr{M}_{d}(k)$ so that $V=K^{d}$ has the structure of a left $U$ module. If

$$
\text { (0) }=I_{0} \subset I_{1} \subset \cdots \subset I_{n}=V
$$

is a composition series of left $U$ modules, then $J \subset\left(I_{j-1}: I_{j}\right)$ for each $1 \leqslant j \leqslant n$. Relative to a basis for $V$ compatible with $(*)$, the matrices in $U$ can be simultaneously brought to block triangular form

$$
U \cong\left[\begin{array}{cccc}
R & & & \\
& & \mathrm{X} & \\
& 0 & & \\
& & & R_{n}
\end{array}\right]
$$

where $R_{j} \cong M_{d_{j}}(K), S \cong \prod_{j-1}^{m} R_{i j}$ for some $m \leqslant n$, and $J$ has its image in strictly upper triangular matrices.

The following examples indicate that there is a relationship between the "distribution" of the $R_{i}$ along the diagonal and the "density" of $J$ which bears on the question of whether there is an algebra structure on $V$ for which $U \cong \mathscr{M}(V)$.

$$
\begin{aligned}
& U \cong\left[\begin{array}{lll}
a & 0 & c \\
0 & a & 0 \\
0 & 0 & b
\end{array}\right], \\
& S \cong K \Pi K \cong\left[\begin{array}{lll}
a & 0 & 0 \\
0 & a & 0 \\
0 & 0 & b
\end{array}\right], \\
& J \cong\left[\begin{array}{lll}
0 & 0 & c \\
0 & 0 & 0 \\
0 & 0 & 0
\end{array}\right] .
\end{aligned}
$$

The following argument shows that $U$ is not isomorphic to the multiplication algebra of any three dimensional algebra.

Suppose $V=K e^{1}+K e^{2}+K e^{3}$ has an algebra structure with $U \cong \mathscr{M}(V)$. Then $V$ has a $U$ composition series

$$
(0)=I_{0} \subset I_{1}=K e^{1} \subset I_{2}=K e^{1}+K e^{2} \subset I_{3}=V,
$$


which is a composition series of ideals. Consider the matrix representation of $\lambda_{e^{1}}$. Since $\lambda_{e^{1}} \in\left(I_{1}: A\right)$, we have

$$
\lambda_{e^{1}}=\left[\begin{array}{lll}
0 & 0 & \alpha \\
0 & 0 & 0 \\
0 & 0 & 0
\end{array}\right] .
$$

Thus $e^{1} e^{3}=\alpha e^{1}$, or

$$
\rho_{e^{3}}=\left[\begin{array}{ccc}
\alpha & 0 & \beta \\
0 & \alpha & 0 \\
0 & 0 & \gamma
\end{array}\right]
$$

But then $e^{2} e^{3}=\alpha e^{2}$, so that

$$
\lambda_{e^{2}}=\left[\begin{array}{lll}
\delta & 0 & 0 \\
0 & \delta & \alpha \\
0 & 0 & 0
\end{array}\right]
$$

This forces $\alpha=0$. Similarly

$$
\rho_{e^{1}}=\left[\begin{array}{lll}
0 & 0 & 0 \\
0 & 0 & 0 \\
0 & 0 & 0
\end{array}\right]
$$

Consider

$$
\lambda_{e^{2}}=\left[\begin{array}{lll}
\alpha & 0 & \beta \\
0 & \alpha & 0 \\
0 & 0 & 0
\end{array}\right]
$$

We have $e^{2} e^{1}=\alpha e^{1}$. But

$$
\rho e_{1}=\left[\begin{array}{lll}
0 & 0 & 0 \\
0 & 0 & 0 \\
0 & 0 & 0
\end{array}\right]
$$

forces $\alpha=0$. Furthermore, $e^{2} e^{3}=\beta e^{1}$ implies

$$
\rho e_{3}=\left[\begin{array}{lll}
\alpha & \beta & \gamma \\
0 & \alpha & 0 \\
0 & 0 & \delta
\end{array}\right],
$$

and thus

$$
\lambda e_{2}=\left[\begin{array}{lll}
0 & 0 & 0 \\
0 & 0 & 0 \\
0 & 0 & 0
\end{array}\right]
$$

Similarly,

$$
\rho e_{2}=\left[\begin{array}{lll}
0 & 0 & 0 \\
0 & 0 & 0 \\
0 & 0 & 0
\end{array}\right] .
$$

This leaves $e^{3} e^{3}=\alpha e^{1}+\beta e^{3}$ as the only possible nonzero product. But $U$ is not generated by

$$
\left[\begin{array}{ccc}
0 & 0 & \alpha \\
0 & 0 & 0 \\
0 & 0 & \beta
\end{array}\right] \text { and }\left[\begin{array}{ccc}
1 & 0 & 0 \\
0 & 1 & 0 \\
0 & 0 & 1
\end{array}\right]
$$


(4.6) The algebras

$$
U_{1}=\left[\begin{array}{lll}
a & b & c \\
0 & a & 0 \\
0 & 0 & d
\end{array}\right] \text { and } U_{2}=\left[\begin{array}{ccc}
a & 0 & b \\
0 & a & c \\
0 & 0 & d
\end{array}\right]
$$

are both multiplication algebras of three dimensional algebras. $U_{1}=\mathscr{M}\left(A_{1}\right)$, where $A_{1}=\sum_{i=1}^{3} K e^{i}$ and

$$
e^{1} e^{2}=e^{1}, \quad e^{2} e^{1}=e^{1}, \quad e^{2} e^{2}=e^{2}, \quad e^{3} e^{3}=e^{1} .
$$

All other products are zero. $U_{2}=\mathscr{M}\left(A_{2}\right)$, where $A_{2}=\sum_{i=1}^{3} K e^{i}$ and

$$
e^{1} e^{3}=e^{1}, \quad e^{2} e^{3}=e^{2} .
$$

All other products are zero.

The crucial ingredients in (4.6) missing from (4.5) are sufficient nonzero products to pick up either idempotent

$$
\left[\begin{array}{lll}
1 & 0 & 0 \\
0 & 1 & 0 \\
0 & 0 & 0
\end{array}\right] \text { or }\left[\begin{array}{lll}
0 & 0 & 0 \\
0 & 0 & 0 \\
0 & 0 & 1
\end{array}\right]
$$

An analysis of multiplication algebras based on this idea is in progress [3].

The author wishes to thank the referee for a very careful reading of the manuscript and for many helpful suggestions and corrections to the original.

\section{REFERENCES}

1. A. A. Albert, The radical of a nonassociative algebra, Bull. Amer. Math. Soc. 48 (1942), 891-897.

2. Scott M. Farrand, The multiplication algebra of a nonassociative algebra, Thesis, U.C.S.D., 1981.

3. S. M. Farrand and D. R. Finston, The multiplication algehra and multiplication ideal of an algebra without nilpotent elements, manuscript.

4. D. R. Finston, The algebra of polynomial functions on a nonassociative algebra, Thesis, U.C.S.D., 1983.

5. I. N. Herstein, Noncommutative rings, Carus Math. Monographs, Math. Assoc. Amer., 1968.

6. N. Jacobson, A note on nonassociative algebras, Duke Math. J. 3 (1937), 544-548.

7. G. N. Müller, Nicht associative separable Algebren über Ringen, Abh. Math. Sem. Univ. Hamburg 40 (1974), 115-131.

8. H. Röhrl, On the zeros of polynomials over arbitrary finite dimensional algebras, Manuscripta Math. 25 (1978), 359-390.

9. R. Wisbauer, Radikale von separablen Algebren über Ringen, Math. Z. 139 (1974), 9-13,

Department of Mathematical Sciences, Virginia Commonwealth University, 1015 West Main STREet, Richmond, Virginia 23284 